\title{
Temperature-stable dielectric TM010-mode resonator and its application to compact base station filter
}

\author{
Toshio Ishizaki ${ }^{a}$ \\ Panasonic Corporation \\ 1 Kotari-yakemachi, Nagaokakyo, Kyoto 617-8520, Japan \\ a) ishizaki.toshio@jp.panasonic.com
}

\begin{abstract}
Instead of a bulky TE-mode resonator with high $\mathrm{Qu}$, a compact TM-mode resonator with moderate $\mathrm{Qu}$ is proposed. The contact between the ceramic rod and the metal cavity is a key point for the filter reliability. Elasticity of the contact portion is well utilized in this case to keep the smooth electrical contact. Also, temperature coefficient of TM-mode resonator is studied. The requirements for characteristics of the ceramic materials are a little different for TE-mode and TM-mode. Finally, high-reliable and temperature-stable TM-mode resonator has been developed. Its application to compact base station filter is demonstrated as well.
\end{abstract}

Keywords: TM-mode, resonator, bandpass filter, reliability, temperature coefficient

Classification: Microwave and millimeter wave devices, circuits, and systems

\section{References}

[1] Y. Kobayashi and S. Yoshida, "Bandpass filters using $\mathrm{TM}_{010}$ dielectric rod resonators," Proc. IEEE MTT-Symposium, pp. 223-235, 1978.

[2] Y. Kobayashi and H. Furukawa, "Elliptic bandpass filters using $\mathrm{TM}_{010}$ dielectric rod resonators," Proc. IEEE MTT-Symposium, pp. 353-356, 1986.

[3] T. Nishikawa, K. Wakino, and Y. Ishikawa, " $800 \mathrm{MHz}$ band channel dropping filter using $\mathrm{TM}_{010}$ mode dielectric resonator," Proc. IEEE MTTSymposium, pp. 199-201, 1984.

[4] T. Nishikawa, K. Wakino, H. Wada, and Y. Ishikawa, "800 MHz band dielectric channel dropping filter using $\mathrm{TM}_{110}$ triple mode resonance," Proc. IEEE MTT-Symposium, pp. 289-292, 1985.

[5] Y. Ishikawa, J. Hattori, M. Andoh, and T. Nishikawa, "800 MHz high power duplexer using TM dual mode dielectric resonators," Proc. IEEE MTT-Symposium, pp. 1617-1620, 1992.

[6] T. Yamakawa and T. Ishizaki, "Dielectric resonator, dielectric filter, and method of supporting dielectric resonance element," United States Patent, US7,106,152, Sept. 2006. 
[7] M. Hoeft, "Bandpass filter using TM-mode dielectric rod resonators with novel input coupling," Proc. IEEE MTT-Symposium, pp. 1601-1604, 2009.

\section{Introduction}

For base station filter, bulky $\mathrm{TE}_{01 \delta}$-mode resonators are commonly used so far due to their very high $\mathrm{Qu}$ values. However, compact base station filters are strongly expected according to the cell sizes of base station cover-areas become smaller. $\mathrm{TM}_{010}$-mode resonator is well-known as it has a compact size and moderate $\mathrm{Qu}[1,2]$. However, there are some difficulties to use as a base station filter with high reliability. Some studies use ceramic shielding cavity which has the same thermal expansion coefficient with the ceramic rod to overcome mechanical stress $[3,4,5]$. This makes the structure of TM-mode resonator very complex and results in high cost.

A novel rod supporting structure which is the same with that of this letter had been shown by the author's US patent [6] and then by Dr. M. Hoeft in [7] again. But, the reliability and the temperature coefficient were not discussed.

The special purposes of this study are to show the results of the reliability test of new TM-mode resonator and to discuss the required temperature coefficient of ceramic material quantitatively.

First in Section 2, basic principle of TM-mode resonator is described. Then the drawbacks of conventional structure are explained.

In Section 3, the new structure and the results of reliability test are illustrated with experimental data.

In Section 4, the temperature coefficient, which is different from TE-mode, is discussed quantitatively.

Then in Section 5, an experimental base station filter, to which the new TM-mode resonators are applied, is shown.

\section{Basics of TM-mode resonator}

Here, the $\mathrm{TM}_{010}$-mode in cylindrical coordinate system is used in this study. Figure 1 (a) shows the structure of TM-mode resonator of either conventional or newly developed ones. The only difference is that the both ends of the ceramic rod are metalized and soldered with metal cavity for conventional one and the rod is mechanically supported with elastic cover portion for newly developed one as shown in the circle of Fig. 1 (a).

The electrical field exists in the rod vertically. The magnetic field surrounds the rod horizontally as a curl. Thus the high frequency current flows from the metal on the both ends of the rod to the metal cavity. The current continuity is very important for stabilizing the resonant frequency and keeping high Qu.

The resonant frequency is determined by the diameters of ceramic rod and metal cavity. The height of the cavity, or the length of the rod, does not 
affect on the resonant frequency, while the height affects on $\mathrm{Qu}$ value of the resonator.

The resonator is coupled electrically with input/output probe or coupled magnetically with input/output loop.

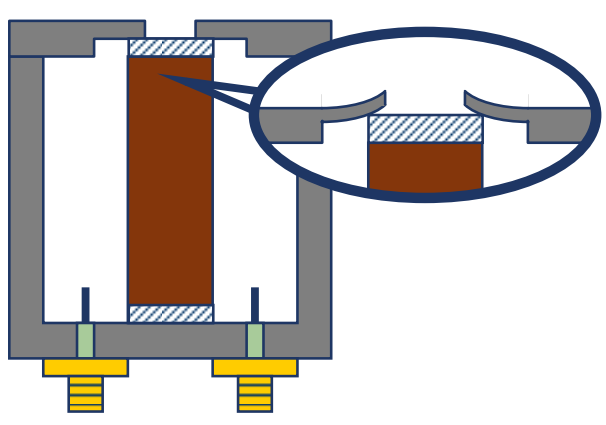

Structure of TM-mode resonator Conventional with soldering New structure (in the circle)

(a)

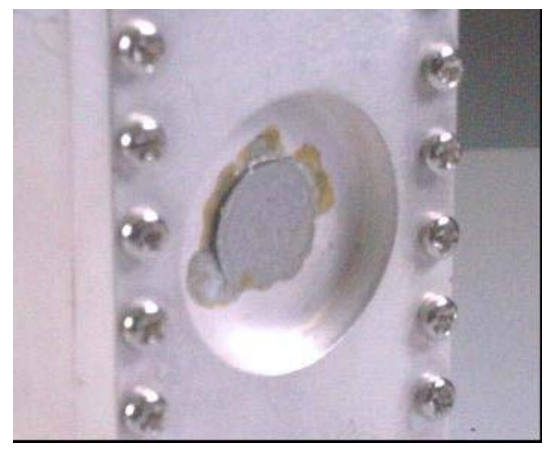

Heat shock test after 1200 cycles between -40 and 80 degrees Celsius Crack is observed between solder and metal (b)

Fig. 1. Structure of TM-mode resonator and result of heat shock test

Here, the performance is compared with those of TEM-mode coaxial resonator and TE-mode cavity resonator at the $2 \mathrm{GHz}$ band. Regarding the volumes and unloaded $\mathrm{Q}$ values, the volume of ceramic TEM-mode coaxial resonator is about $2 \mathrm{cc}$ with $\mathrm{Qu}$ less than 3,000, and that of ceramic TE-mode cavity resonator is about $70 \mathrm{cc}$ with Qu more than 14,000, while that of ceramic TM-mode resonator is about $5 \mathrm{cc}$ to $15 \mathrm{cc}$ with $\mathrm{Qu}$ of 3000 to 8000 . So the features of TM-mode resonator are the very compact size and the moderate $\mathrm{Qu}$, which are suitable for compact base station application.

The following parameters are used in the simulations and the measurements. The diameter of the rod is $9 \mathrm{~mm}$ and the dielectric constant of the ceramics is 42 . The cavity size is $22 \mathrm{~mm}$ by $22 \mathrm{~mm}$ by $30 \mathrm{~mm}$.

For conventional TM-mode resonator, the both ends of rod are metalized with silver and usually fixed by soldering to the cavity. It might not be bad to obtain high $\mathrm{Qu}$, if did not consider the reliability. The problem is the mechanical stress due to the difference of thermal expansion coefficients between ceramic and metal. As shown in Fig. 1(b), a crack was observed between solder and metal after 1200 cycle heat shock test. The temperature is rapidly changed from -40 and +80 degrees Celsius then the opposite direction in an iterative way.

\section{New structure and reliability test}

The proposed new structure of TM-mode resonator is also shown in the circle of Fig. 1 (a) as mentioned. The elastic cover portion supports the rod mechanically. Owing to this novel structure, the support becomes crack-free. 
Actually, the author tested varieties of structures, but this was the best one of those.

Table I shows the result of the reliability test. The test condition of heat shock is from -40 to +80 degrees Celsius and 100 cycles. As the results, the frequency difference is $0.262 \mathrm{MHz}$ and $\mathrm{Qu}$ difference is -1087 for the conventional structure, while the frequency difference is $-0.059 \mathrm{MHz}$ and $\mathrm{Qu}$ difference is -220 for the proposed new structure. The result of the new structure is drastically better. For the conventional structure, some mechanical destruction might be under going at the support portion. Thus, the remarkable $\mathrm{Qu}$ degradation was observed.

Table I. Result of the reliability test

\begin{tabular}{|c|c|c|c|c|c|c|}
\hline \multirow{2}{*}{} & \multicolumn{2}{|c|}{ Before } & \multicolumn{2}{c|}{ After } & \multicolumn{2}{c|}{ Difference } \\
\cline { 2 - 7 } & $\mathrm{f}_{\mathrm{c}}[\mathrm{MHz}]$ & $\mathrm{Q}$ & $\mathrm{f}_{\mathrm{c}}[\mathrm{MHz}]$ & $\mathrm{Q}$ & $\Delta \mathrm{f}[\mathrm{MHz}]$ & $\Delta \mathrm{Q}$ \\
\hline $\begin{array}{c}\text { Conventi } \\
\text { onal }\end{array}$ & 2200.997 & 5,252 & 2201.259 & 4,165 & 0.262 & $-1,087$ \\
\hline This work & 2194.357 & 5,489 & 2194.298 & 5,269 & -0.059 & -220 \\
\hline
\end{tabular}

\section{Temperature coefficient of TM-mode}

Next, let's consider the temperature coefficient of TM-mode resonator.

The cavity is made by copper with silver-plated in this case. The lengthexpansion coefficient of copper is 16.5 [ppm/degree] and that of the ceramics is 5 [ppm/degree]. The frequency drift from -40 to +80 [degree Celsius] is $-2.2 \mathrm{MHz}$. The simulation and the measurement for frequency drift show very good agreement. Thus, more positive temperature coefficient of ceramics

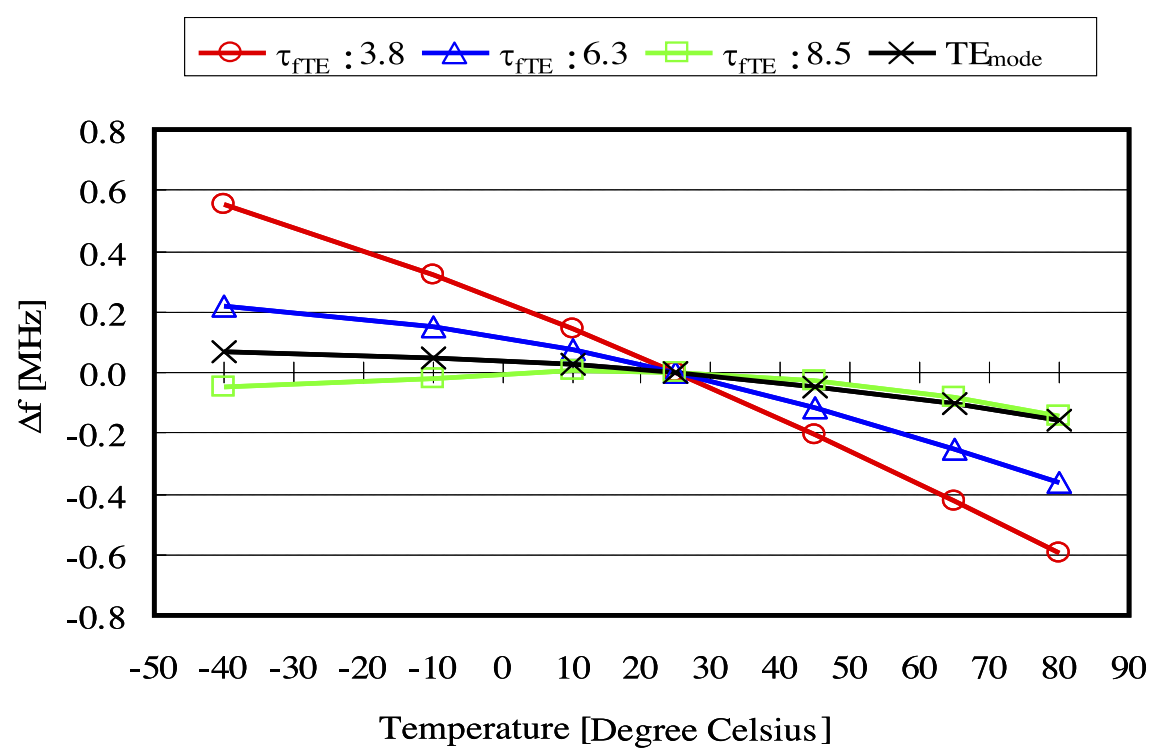

Fig. 2. Frequency drift and temperature coefficient 
is required for TM-mode comparing TE-mode. In other words, temperature coefficients of zero frequency drift are actually different for TM-mode and TE-mode based on the mechanisms.

As you may know very well, the temperature coefficient of TE-mode resonator is described as that of $\mathrm{TE}_{01 \delta}$-mode resonator in the super-invar cavity with enough gap distances above and below the ceramics. The coefficient depends on the temperature coefficient of permittivity of the ceramics and the thermal expansion coefficient. However, please consider the basic equations of resonances. They are quite different for TE-mode and TM-mode. It's easy to understand the most adequate temperature coefficients $\tau_{\text {fTE }}$ and $\tau_{\text {fTM }}$ are different indeed.

Fig. 2 shows the relation between frequency drift and temperature coefficient in terms of temperature variation obtained by measurements.

In Fig. 2, the red line of $\tau_{\mathrm{fTE}}=3.8$ means that the ceramic material with temperature coefficient of 3.8 [ppm/degree] for TE-mode resonator is adopted for the rod of TM-mode resonator and measured as TM-mode resonator. Next, the blue line of $\tau_{\mathrm{fTE}}=6.3$ was measured. Then, by extrapolation, we can obtain $\tau_{\text {fTE }}=8.5$ as the best temperature coefficient for TM-mode. Then the performance was measured. All lines in Fig. 2 are measured data and show temperature characteristics for TM-mode resonators except for line of $\mathrm{TE}_{\text {mode }}$ (black line with cross marks). Only this data is for TE-mode resonator with zero [ppm/degree] ceramic material for TE-mode resonator.

The result is that the $\tau_{\text {fTE }}$ of $8.5\left[\mathrm{ppm} /\right.$ degree] corresponds to $\tau_{\text {fTM }}$ of zero [ppm/degree]. It means that the ceramics of temperature coefficient of $8.5[\mathrm{ppm} /$ degree] for TE-mode resonator should be used for the rod of TM-mode resonator with temperature coefficient of zero [ppm/degree]. Therefore the high temperature stable resonator can be obtained for TM-mode, and the performance is almost the same with that of TE-mode.

Again, the temperature coefficient of TM-mode resonator is not inferior to that of TE-mode. Although there might be some widespread misunderstanding for TM-mode, this work makes clear that the temperature coefficient of TM-mode can be optimized to be zero.

\section{Experimental filter}

An experimental filter was developed for W-CDMA transmitter filter application. The pass band is $2130 \mathrm{MHz}$ to $2150 \mathrm{MHz}$. The number of pole is four.

A TE-mode filter was also made for comparing purpose. For TE-mode filter, loss is $0.3 \mathrm{~dB}, \mathrm{Qu}$ is 8000 , and volume is $250 \mathrm{cc}$. For TM-mode filter, loss is $0.65 \mathrm{~dB}, \mathrm{Qu}$ is 3500 , and volume is $100 \mathrm{cc}$. The $\mathrm{Qu}$ values are somewhat degraded comparing with resonator alone due to some tuning mechanism. The volume is only one-thirds of the TE-mode filter.

Fig. 3 shows the frequency responses of both filters. The pass band performances around $2.1 \mathrm{GHz}$ are almost the same. On the other hands, the spurious responses in the frequency range from $2.4 \mathrm{GHz}$ to $3.5 \mathrm{GHz}$ are quite 


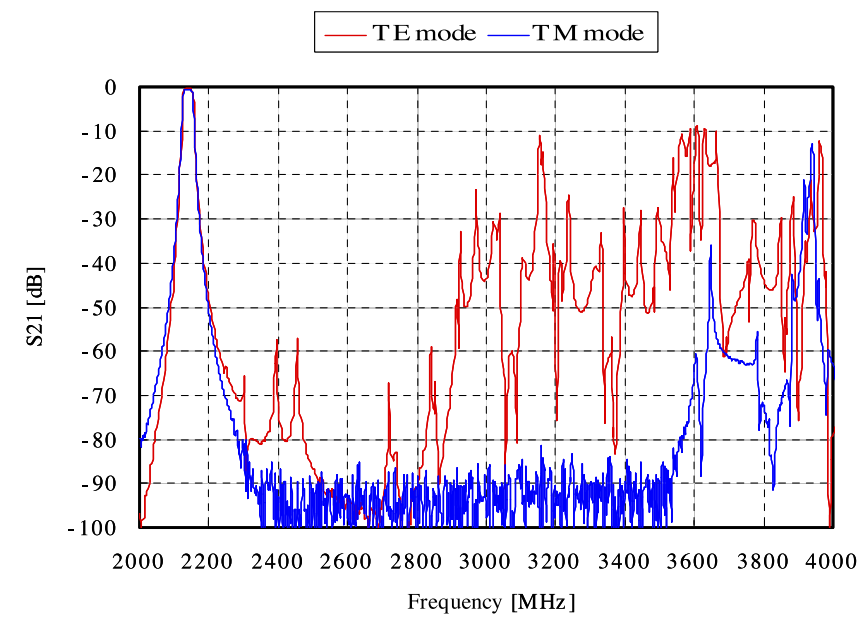

Fig. 3. Spurious performance of both filters

different. For the TM-mode filter, it is owing to the second spurious resonance mode, which is far away from the lowest $\mathrm{TM}_{010}$ resonance mode. For the TE-mode filter, there are many spurious resonance modes, such as hybrid modes, in the vicinity of the main $\mathrm{TE}_{01 \delta}$ resonance mode. Apparently, TM-mode filter is better than TE-mode filter.

\section{Conclusion}

The problem of conventional TM-mode filter was pointed out. The crack at the supporting connection was removed by adopting new structure, which utilized the elasticity of the cover portion. The result of heat cycle test showed high reliability of this structure. The optimum temperature coefficient was determined by experiments for the ceramic rod of TM-mode filter quantitatively. It was equal to 8.5 [ppm/degree] expressed by TE-mode temperature coefficient. Experimental 4-pole filter was demonstrated as well. The insertion loss was $0.65 \mathrm{~dB}$ and the volume was only one-thirds of TE-mode filter. In addition, the spurious response of TM-mode filter was superior to that of TE-mode filter. As a result, the supeority of TM-mode resonator applied to compact base station filter was confirmed. 